CERN LIBRARIES, GENEVA

$\frac{17}{3}$

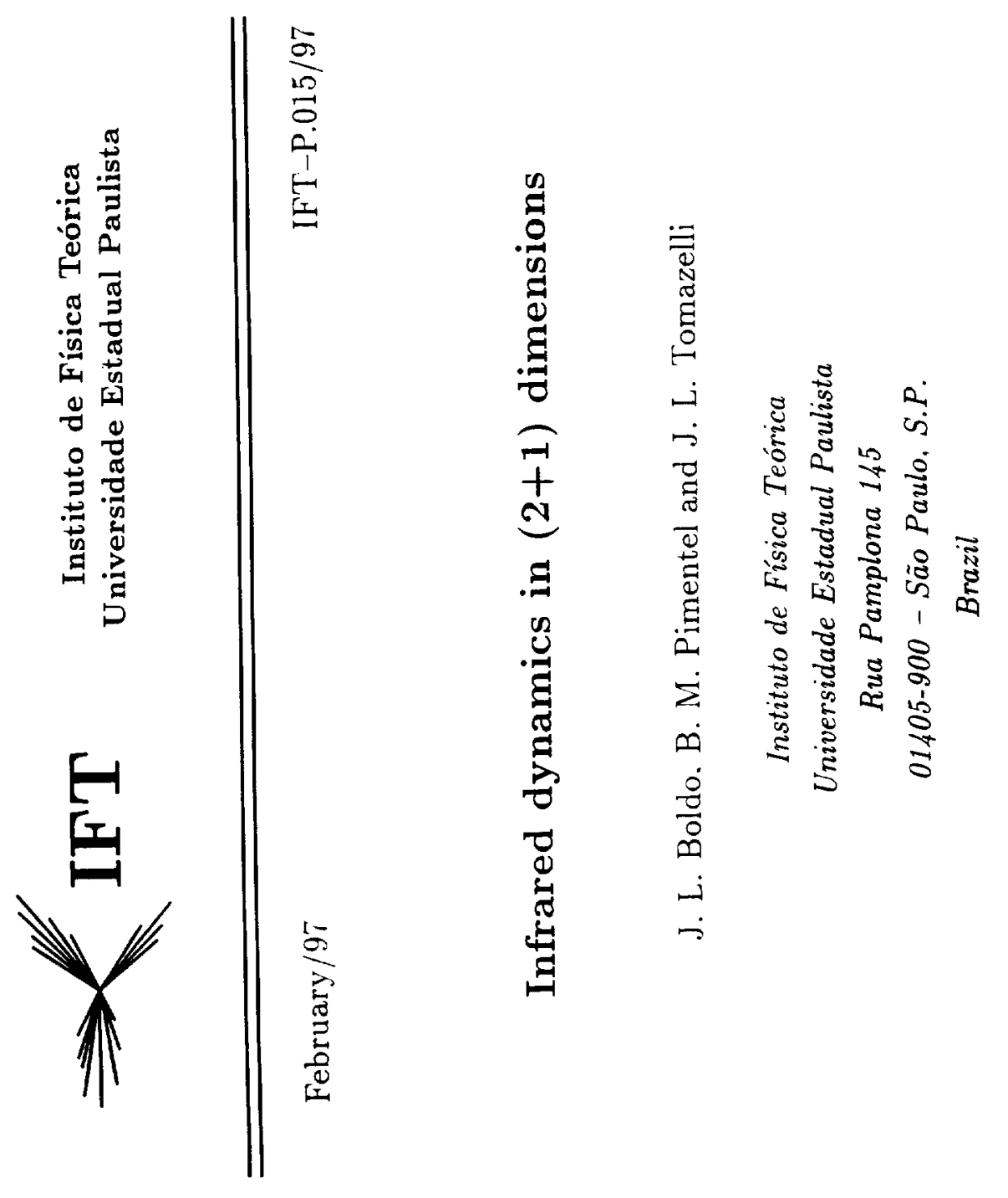




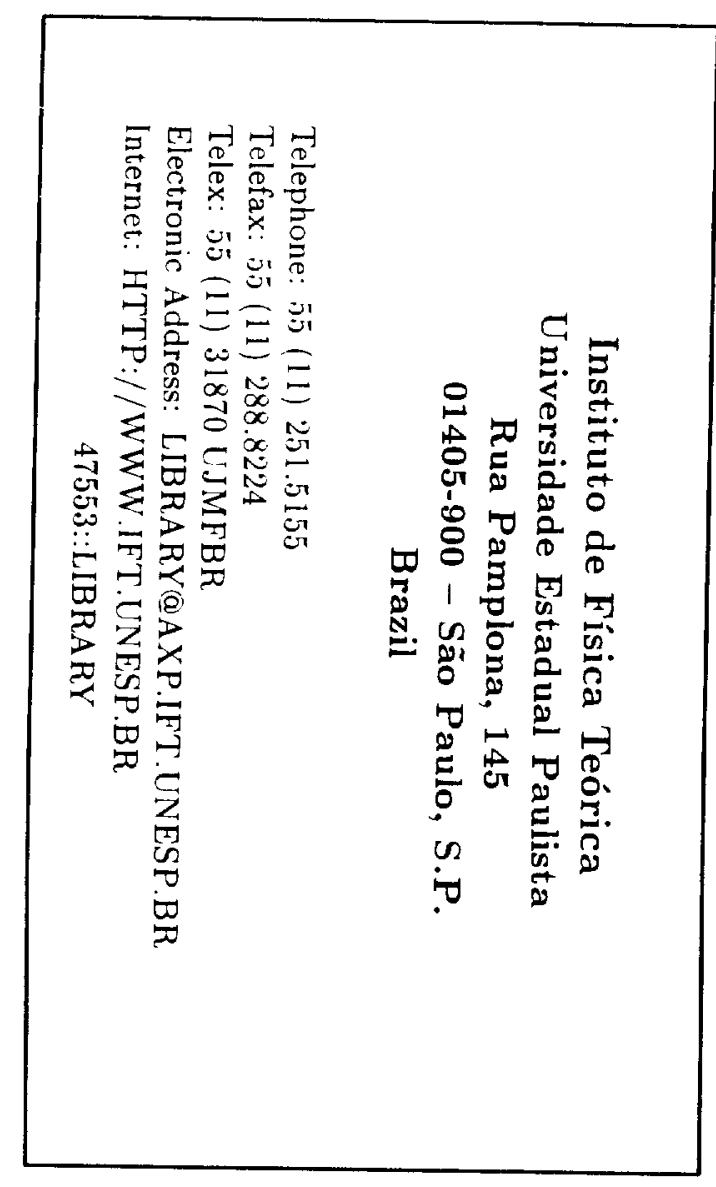




\section{Infrared Dynamics in (2+1) Dimensions}

\author{
J. L. Boldo ${ }^{1}$, B. M. Pimentel ${ }^{2}$ and J. L. Tomazelli ${ }^{3}$ \\ Instituto de Física Teórica \\ Universidade Estadual Paulista \\ Rua Pamplona, 145 \\ 01405-900 - São Paulo, SP - Brazil
}

\section{Abstract}

In this work we study the asymptotic behavior of $(2+1)$-dimensional quantum electrodynamics in the infrared region. We show that an appropriate redefinition of the fermion current operator leads to an asymptotic evolution operator which contains a divergent Coulomb phase factor and a contribution of the electromagnetic field at large distances, factored from the evolution operator for free fields, and conclude that the modified scattering oprator the spaces of coherent states of the electrognetic field, as in the Kulish-Faddeev model for QED in four space-time dimensions.

\section{Introduction}

In QED the method to deal with non-stationary processes as electron scattering by an external potent with the emis ding $S$ matrix expansion in powers of $e^{2}$, leads to the so-called infrared divergences in the probability amplitudes for such processes.

The pioneering program of taking into account the collective effect of low-energy photons was accomplished by Bloch and Nordsieck ${ }^{[1]}$ in the late 30 's. They showed that the probability of emission of a finite number of low-energy photons is zero instead of infinite, as predicted by perturbation theory. On the other hand, when the transition probability is extended to all by perturbation theory. On the other hand

possible final states one gets a finite result. which can be compared with the experimental data, besides the ill definition of the scattering operator, where the asymptotic dynamics is not taken into account. Murota ${ }^{[2]}$ was the first to incorporate the exact contribution of low-energy photons in the definition of the $S$ operator. incorporate the exact contribution of low-energy photons in the definition of the 5 . Later Kulish and Faddeev ${ }^{[3]}$ proposed in addition to the redefinition of this operator a modified
space of asymptotic states. The Kulish-Faddeev model is based on early arguments, mainly

'Supported by CAPES

${ }^{2}$ Partially supported by CNPq

s Supported by FAPESP those of Kibble[4], where the fork space representation of the llillert space conlaining a finite number of low-cnergy photons, is replaced by a roherent-state representation for an infinite number of these phetons, in such a way that matris elements of the l)eson s' operat or between these states are finite and nonzero.

The investigation of the infrased problem in four-dimensional gange theories can be extended to the three-dimensional case, where a more careful analysis is repuired ${ }^{\text {lit. }}$. This study can stil be justified if we hear in mind that some physical phenomena, such as the quantum Hall effect and the high $T_{c}$ superconductivity. can be better unclerstood in the context of quantum electrodynamios in $(2+1)$ dimensions.

This paper is organized as follows: in the next 1 wo sertions (2 and 3 ), we discuss the applicability of the usual S-operator formalism and present a plysical motivation for modifying the wave operators in the non-relativistic ('onlomb scattering of a charged particle: section 4 is devoted to the construction of the asymptotir coolution operator for $\mathrm{QED}_{3}$. and in section 5 we summarize our results anrl comelusions.

\section{The S Operator Formalism}

Consider a quantum system described lyy a Ilamiltonian $/ /$ constituted by two terms

$$
H=H_{\mathrm{n}}+1(1)
$$

where $U_{0}$ is the free Ilamiltonian and $l(t)$ is a shopt-range interaction potent ial

$$
\lim _{1 \rightarrow \pm \infty} l(1)=0 \text {. }
$$

This implies that asymptotically the scalteged particles are supposed to be free. Thus, since in scattering processes the particles are observed only in asyuntotic regions $(l \rightarrow \pm x)$, the asymptotic states are cigenstates of $H_{11}$.

Using these ideas, one defines the sionerator in perturloation theory assuming that the asymptotic dyouniss is given by $H_{0}$

$$
r=1 H_{i n+1}^{+} 1 H_{i n}
$$

where $W_{\text {in }}$ are the wave operators

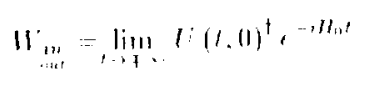

and

$$
l(t, s)=e^{-i n(t-s)} .
$$

Using (5), one rewrites the $S$ matrix as

$$
\varphi=\lim _{\substack{t \rightarrow+\infty \\ \rightarrow \rightarrow-\infty}} i^{i H_{0} t}(1, s) c^{-i H_{0} s} .
$$

From this definition the physical meaning of the $S$-matrix is the following: an asymptotic initial From this defition the prom state $\varphi$, taken at $t=0$, is evolved to $s \rightarrow-\infty$ 
$-\infty$ to $t \rightarrow+\infty$ via $U(t, s)$ and, finally, from $t \rightarrow+\infty$ to $t=0$ through free dymamics. The transition probability from this state to another final asymptotic state $\psi$ is given by

$$
|\langle\psi|S| \varphi\rangle|^{2}
$$

where the asymptotic states belong to the Fock space.

Solving the equation of motion for the time evolution operator, the $S$-matrix can be rewritten as a time-ordered product

$$
S=T \exp \left\{-i \int_{-\infty}^{+\infty} V^{\prime}(t) d t\right\}
$$

where $V^{I}(t)$ é is the potential in the interaction picture. If the interaction potential vanishes in remote regions, such that

$$
\int_{-\infty}^{+\infty} d s\left\|V^{I}(s)\right\|<\infty
$$

then the Dyson series for the $S$ operator (8) is alsolutely convergent. Thus, the $S$ operator is unitary in the Fock space, provided the particles have free dynamics in asymptotic regions.

The above method is not suitable to describing scattering processe's when we consider longrange potentials such as the Coulomb potential. In this case, even at far regions this potential cannot be neglected.

\section{Non-relativistic Coulomb Scattering}

In order to illustrate the method employed in the construction of the asymptotic operator $U_{a s}(t)$ let us consider the scattering of a charged particle by a Coulomb potential in two dimensions The Hamiltonian of the system has the following form:

$$
H=\frac{\vec{p}^{2}}{2 m}+g \ln r=H_{0}+V
$$

where $m$ is the mass of the scattered particle and $g$ is the product of the charges of the particle and the scattering center.

First of all one constructs the potential sliape in the asymplotic region in the interaction picture and then one obtains the wave packet which will represent the scattered particle in this region. For this purpose one considers the observables $\vec{x}$ and $\vec{p}$ as the position and momentum operators in the interaction picture. In this representation these operators satisfy the following equations of motion:

$$
\frac{d O_{I}}{d t}=\frac{1}{i}\left[O_{I}, H_{0}\right]
$$

Using the Hamiltonian (10) and the above equation, we see that the momentum of the scattered particle is a constant of motion:

$$
\frac{d \vec{p}}{d t}=\frac{1}{i}\left[\vec{p}, H_{0}\right]=0
$$

Similarly, the cquation of metion for the coordinates

$$
\frac{d \vec{x}}{d t}=\frac{1}{i}\left[\vec{r}, H_{0}\right]=\frac{\vec{p}}{m}
$$

whose solution

$$
\vec{x}(t)=\vec{x}+\frac{\vec{p}}{m} t
$$

which describes the time evolution of the coordinate operator of the scattered particle, is identical to the classical trajectory of a particle in uniform rectilinear motion. Ftom these results one can obtain the shape of the interaction potential at large distances, assuming that in this region the particles behave as classical particles with well defined trajectories. Thus, for $|t| \rightarrow \infty$

$$
r(t)=g \ln \left(\frac{|\vec{p}|}{m} t\right)
$$

This potential, which describes the interaction in the asymptotic region $|t| \rightarrow \infty$, is not absolutely convergent and its contribution to the asymptotic dynamics cannot be underestimated. In other words, the asymptotic dynamiss is not governed loy $H_{0}$ hut by the operator

$$
U_{a s}(t)=U_{0}+V_{a s}(t)=U_{0}+g \ln \left(\frac{|\vec{p}|}{m}\right)
$$

With this Hamiltonian describing the asymptotic dynamics and taking into account that $V_{a s}(t)$ in the interaction picture is the same as in the Schrölinger picture, since

$$
\left[V_{a s}(t), H_{0}\right]=0 \text {, }
$$

the wave function which describes the behavior of the particle in this region is oblained by solving the Schrödinger equation for $H_{r s}$

$$
i \frac{d}{d t}|r, 1\rangle=I_{a s}(1)|a, 1\rangle
$$

where $|\alpha, t\rangle$ is the physical state of the scattered particle. In the momentum representation the above equation becomes

$$
i \frac{d}{d t} \Psi(\vec{p}, t)=\left[\frac{\vec{p}^{2}}{2 m}+g \ln \left(\frac{|\vec{p}|}{m} t\right)\right] \Psi(\vec{p}, t),
$$

whose solution is

$$
\begin{aligned}
\Psi(\vec{x}, t)= & \frac{1}{2 \pi} \int d \vec{p} \Psi(\vec{p}, t) e^{i \vec{p} \cdot \vec{x}} \\
= & \frac{1}{2 \pi} \int d \vec{p} e^{i \vec{p} \cdot \vec{x}} c(\vec{p}) \\
& \times \exp \left\{-i \frac{\vec{p}^{2}}{2 m} t-i g\left[t \ln \left(\frac{|\vec{p}|}{m} t-1\right)-t_{0} \ln \left(\frac{|\vec{p}|}{m} t_{0}-1\right)\right]\right\} .
\end{aligned}
$$


The choice of this solution is due to certain initial conditions for equation (19). These are determined by considering that the time variation of the coordinate and momentum distributions for the particle represented by the wave packet (20) for $|t| \rightarrow \infty$ must be governed by the classical dynamics.

The wave packet (20) can be written as

$$
\begin{aligned}
\Psi(t) & =U_{a q}(t) \Psi \\
& =e^{-i H_{0} t} \exp \left\{-i g\left[t \ln \left(\frac{|\vec{p}|}{m} t-1\right)-t_{0} \ln \left(\frac{|\vec{p}|}{m} t_{0}-1\right)\right]\right\} \Psi .
\end{aligned}
$$

The previous example shows that the choice of the Hamiltonian which describes the asymptotic dynamics depends on the physical origin of the problem, in contrast with the usual definitions in the formal theory of scattering, where $H_{0}$ is taken as the asymptotic operator in the wave operators.

We then redefine the wave operators in the following way:

$$
W_{\text {out }}^{\text {in }}=\lim _{t \rightarrow \mp \infty} e^{-i H t} U_{a s}(t) .
$$

\section{The Asymptotic Operator in $\mathrm{QED}_{3}$}

Now, we can construct the asymptotic operator $\mathrm{U}_{a s}(\mathrm{t})$ in quantum electrodynamics. In order to describe a system of interacting electrons, positrons and photons we shall use $\Psi$ and $\bar{\Psi}$ for the operators of the electron-positron field and $\mathrm{A}^{\mu}$ for the operator of the electromagnetic field. These field operators can be expressed in terms of the creation and annihilation operators as follows:

$$
\begin{gathered}
A^{\mu}(x)=\frac{1}{2 \pi} \int \frac{d \vec{k}}{\sqrt{2 \omega_{k}}}\left(a^{\mu}(\vec{k}) e^{-i k \cdot x}+a^{\mu \dagger}(\vec{k}) e^{i k \cdot x}\right), \\
\Psi(x)=\Psi^{(+)}(x)+\Psi^{(-)}(x) \\
\Psi(x)=\bar{\Psi}^{(+)}(x)+\bar{\Psi}^{(-)}(x)
\end{gathered}
$$

where

$$
\begin{aligned}
& \Psi^{(+)}(x)=\frac{1}{2 \pi} \int d \vec{p} \sqrt{\frac{m}{p_{0}}} \sum_{r} b_{r}(\vec{p}) u_{r}(\vec{p}) e^{i \vec{p} \cdot \vec{x}-i p_{0} t} \\
& \Psi^{(-)}(x)=\frac{1}{2 \pi} \int d \vec{p} \sqrt{\frac{m}{p_{0}}} \sum_{r} d_{r}^{\dagger}(\vec{p}) v_{r}(\vec{p}) e^{-i \vec{p} \cdot \vec{x}+i p_{0} t} \\
& \Psi^{(-)}(x)=\frac{1}{2 \pi} \int d \vec{p} \sqrt{\frac{m}{p_{0}}} \sum_{r} b_{r}^{\dagger}(\vec{p}) \bar{u}_{r}(\vec{p}) e^{-i \vec{p} \cdot \vec{x}+i p_{0} t}
\end{aligned}
$$

$$
\bar{\Psi}^{(+)}(r)=\frac{1}{2 \pi} \int d \vec{p} \sqrt{\frac{m}{p_{0}}} \sum_{r} d_{r}(\vec{p}) \vec{r}_{r}(\vec{p}), \overrightarrow{v^{\prime} \cdot \vec{r}-i p_{0} !}
$$

and $a^{\mu}(\vec{k}), b_{r}(\vec{p})$ and $d_{r}(\vec{p})$. are antilitalion operators of photons, electrons and positrons. respectively.

The interaction operator of the system of photons and charged particles, in the interaction representation, is given by

$$
l_{i}=\int j^{\prime \prime}(x) \cdot l_{\mu}(x) d \vec{x}
$$

where $j^{\prime \prime}(x)$ is the current operator

$$
j^{\prime \prime}(x)=c: \bar{\Psi}(x) \gamma^{\prime \prime} \Psi(x): \text {. }
$$

The decomposition of $\Psi(x)$ into a creation part $\Psi^{(-)}(x)$, and an annihilation part $\Psi^{(+)}(x)$, separates $j^{\mu}(x)$ into four terms

$$
\begin{aligned}
& j^{+++}=\left(\bar{\Psi}^{(+)},^{\prime \prime} \Psi^{(+)} .\right. \\
& i^{\prime \prime--}=\left(\pi^{(-)} \gamma^{\mu} \psi^{i-1} .\right. \\
& i^{n+-}-\cdot: \bar{W}^{(+)} ?^{\mu} \Psi^{(-)}: \\
& j^{\prime \prime+}=e: \bar{\psi}^{(-)} \gamma^{\prime \prime} \Psi^{(+)}: \text {. }
\end{aligned}
$$

We can investigate the asymptotic heliavior of this expression for $|t| \rightarrow \infty$. In this limit only $j^{\prime \prime+}$ and $j^{n-t}$ survive. In fart, since $l i(t)$ conlains the time $t$ in the form $f^{\text {int }}$. only those terms for which o approach to zero within the range of integration over monernt um space will survive. Thuss, the terms $j^{\mu--}$ and $j^{\prime \prime++}$ do not contribute in the asymptotic region, since the argument. of the exponential function characterizing 1 la. time dependence of these terms is proportional to

$$
r^{\prime \prime}+q^{\prime \prime} \mp \omega=\left(\vec{p}^{2}+m^{2}\right)^{\frac{1}{2}}+\left((\overrightarrow{\vec{l}}+\vec{k})^{2}+m^{2}\right)^{\frac{1}{2}} \mp k_{0} .
$$

which is different from zero for all $\vec{p}$ and $\vec{k}$. On the other haurl. the terms $j^{\mu+-}$ and $j^{\mu-+}$ have an argument of the exponential proportional to

$$
\eta^{0}-\eta^{\prime \prime} \mp \omega=\left(\vec{p}^{2}+m^{2}\right)^{\frac{1}{2}}-\left((\vec{p}+\vec{k})^{2}+m^{2}\right)^{\frac{1}{2}} \mp k_{0} .
$$

which vanishes for $\vec{k}$ equal to zero and for all $\vec{p}$. 
Other simplifications can be done, taking the fact that the main contribution to the remaining integrals in the limit $|t| \rightarrow \infty$ comes from the neighborhood of sinall momentum $\vec{k}$ i.e.

$$
p^{0}-q^{0} \mp \omega \simeq \mp \frac{p \cdot k}{p^{0}}
$$

and

$$
\bar{u}_{r}(p) \gamma^{\mu} u_{s}(q) \simeq \frac{p^{\mu}}{m} \delta_{r s}
$$

where

$$
\vec{p}+\vec{k}=\vec{q} \text {. }
$$

Thus in $(2+1)$ dimensions, the potential which describes the interaction between electrons and positrons with the electromagnetic field in the asymptotic region is given by

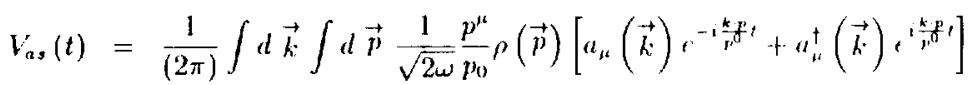

$$
\begin{aligned}
& =\frac{1}{(2 \pi)} \int \frac{d \vec{k}}{\sqrt{2 \omega_{k}}} j_{a s}^{\mu}(\vec{k}, t)\left[a_{\mu}(\vec{k}) e^{-i \omega t}+a_{\mu}^{\dagger}(-\vec{k}) c^{i \omega t}\right],
\end{aligned}
$$

where $\rho(\vec{p})$ is the charge-density operator

and

$$
\rho(\vec{p})=c\left[b_{r}^{\dagger}(\vec{p}) b_{r}(\vec{p})-d_{r}^{\dagger}(\vec{p}) d_{r}(\vec{p})\right]
$$

$$
j_{a s}^{\mu}(\vec{k}, t)=\int p^{\mu} e^{i \frac{\vec{k} \cdot \vec{p}_{p}}{\mathbf{p}^{0}}} \rho(\vec{p}) \frac{d \vec{p}}{p^{0}}
$$

is an operator that has the shape of a current distribution of a particle with charge density $\rho(\vec{p})$ and uniform velocity $\frac{p^{\mu}}{p^{\alpha}}$. In fact, the eigenvalues of the operator (39), acting in a space of charged particles, are classical current densities due to the motion of these particles, showing that, asymptotically, the scattered particles behave as classical particles.

As in the non-relativistic case, the Hamiltonian that describes the asymptotic dynamics is given by

$$
H_{a s}=H_{0}+V_{a s}(t)
$$

where $H_{0}$ is the free Hamiltonian. We can obtain $U_{a s}(t)$ by solving the Schrödinger equation for the time evolution operator

$$
i \frac{d}{d t} U(t)=H(t) U(t)
$$

where $H$ is the Hamiltonian of the system. In our problem $H=H$, and, therefore, the above differential equation must be satisfied by the operator $U_{a s}(t)$. In analogy to the non-relativistic result, we look for solutions of the above equation of the following type:

$$
U_{a s}(t)=e^{-i H_{0} t} Z(t) .
$$

Substituting this operator in equation (4I), we oblain the following equation of motion for $Z(t)$

$$
i \frac{d}{d t} Z(t)=V_{a s}^{l}(t) Z(t)
$$

where $V_{a s}^{I}(t)$ is the asymptotic potential operator in the interaction picture, namely

$$
V_{a s}^{I}(l)=c^{i / H_{0} t} V_{n, s}(l),-i H_{0} t
$$

The solution of the above equation is the time-ordered product

$$
Z(t)=T \exp \left\{-i \int^{t} V_{u s}^{I I}(\tau) d \tau\right\}
$$

whose solution can be simplified if we note that the commutator of potentials at different times is a c-number:

and, consequently,

$$
\left[l_{a s}^{I}\left(t_{1}\right), l_{a s}^{\prime}\left(t_{2}\right)\right]=c-n u m b e r
$$

$$
\left[v_{a s}^{I}(t),\left[V_{a s}^{I}\left(t_{1}\right), v_{\mathrm{a} s}^{I}\left(t_{2}\right)\right]\right]=0, \quad \forall t, t_{1}, t_{2}
$$

Then, the solution for $Z(t)$, with time ordering, can be rewritten as an ordinary product

$$
\begin{aligned}
Z(t) & =\exp \left\{-i \int^{t} V_{a s}^{I}(\tau) d \tau\right\} \exp \left\{-\frac{1}{2} \int_{t_{0}}^{t} d \tau \int_{t_{0}}^{\tau} d s\left[V_{a s}^{\prime}(\tau), V_{a s}^{I}(s)\right]\right\} \\
& =\exp \{R(t)\} \exp \{i \phi(t)\},
\end{aligned}
$$

where

$$
R(1)=-i \int_{t_{0}}^{1} l_{a s}^{i}(\tau) d \tau
$$

and

$$
\phi(t)=-\frac{1}{2 i} \int_{t_{0}}^{t} d \tau \int_{t_{0}}^{\tau} d s\left[V_{a s}^{\prime l}(\tau), l_{u s}^{\prime l}(s)\right]
$$

From this result we see that the asymptotic operator consists of two factors that commute. The first involves photon operators. The second is a plase factor which can be written as

$$
\phi(t)=\frac{1}{4 \pi} \int d \vec{q} \int d \vec{p} \frac{p \cdot q}{p^{(0} q^{0}} p(\vec{p}) \rho(\vec{q}) t\left[1-\ln \left(2\left|\frac{\vec{p}}{p_{0}}-\frac{\vec{q}}{q_{0}}\right| t\right)\right],
$$

showing that this is a relativistic generalization of the above nnentioned Coulomb phase. The eigenvalue of this operator acting in a space of charged particles gives the Coulomb interaction among all the particles of the system.

With $Z(t)$ defined in (48), the asymptotic operator is rewritten as

$$
U_{u s}(t)=e^{-i H_{0} t} e^{R(t)} e^{i \phi(t)}
$$

and, following the non-relativistic generalization, the operator $S$ is expressed as

$$
S=\lim _{\substack{t \rightarrow+\infty \\ s \rightarrow-\infty}}\left(I_{a s}^{\dagger}(t) e^{-i H(t-s)} U_{a s}(s)\right.
$$




\section{Concluding Remarks}

We have seen that the asymptotic dynamics in $\mathrm{QHD}_{3}$ in the infrared region is governed by an evolution operator which contains an infrared phase factor and a factored contribution of the asymptotic electromagnetic field. This leads to definition (53) for the $S$ operator, which differs from the Dyson S-natrix (1) by the replacement.

$$
e^{-i H_{0} t} \rightarrow I_{\text {a. }}(1)
$$

since the infinite range of the conlomb potential destroys the behavior of free dynamies in asymptotic regions. According to kulish and Fadleev, the new $S$ operator maps spaces of coherent states of the electromagnetic field. instead of Fock spaces of initial and finat states of free particles.

Altermatively, one may enbed the asymptotic clynamics in the St operator by perforning an arbitrary splitting of the int craction hanilonian indo $I_{s}+I_{h}$, where $I_{s}$ carries the cont ribution of the soft photons ${ }^{[6]}$.

\section{References}

[1] F. Bloch and A. Nordsieck, Phys. Rev. 52, 54, (1937);

[2] T. Murota, Prog. Theor. Phys. 244, 1109, (1960);

[3] P. Kulish and L. Faddeev, Teor. Mat. Fiz. 4, 153 (1970) [Theor. Math. Phys. 4, 745 (1970)];

[4] T. W. B. Kibble, Phys. Rev. 173, 1527; 174, 1882; 175, 1624 (1968);

[5] B. M. Pimentel and J. L. Tomazelli, J. Phys G, $\underline{20}, 845$ (1994);

[6] J. L. Boldo, B. M. Pimentel and J. L. Tomazelli, in preparation 
